\title{
CYCLIC DEFORMATION OF TUNGSTEN SINGLE CRYSTALS
}

\author{
Keith J. Bowman and Ronald Gibala \\ Department of Materials Science and Engineering, University of Michigan \\ Ann Arbor, Michigan 48109 \\ (Received August 8, 1986)

\section{Introduction}

In the low temperature $\left(T \lesssim 0.20 T_{m}\right)$ monotonic deformation of bcc metals, microstrain is characterized as the plastic strain accommodated by the motion of mobile, non-screw dislocations. After exhaustion of these mobile non-screw dislocations into the screw orientation, higher stresses are required for macroscopic flow by motion of much lower mobility screw dislocations. These differences in dislocation dynamics are manifested in the large temperature and strain rate dependence of macroscopic flow behavior in bcc metals (1). By comparison, the cyclic deformation behavior of bcc metals at low temperatures in terms of this two-dislocation model is not well understood. Part of the problem is that experimental data have thus far been confined to limited investigations on iron, niobium and molybdenum (2-7). For these materials, experiments well into the thermal regime of reduced screw dislocation mobility are difficult in that measurements of deformation behavior are required at temperatures significantly below room temperature or at very high strain rates. Consequently, the low temperature cyclic deformation behavior of bcc metals remains poorly characterized. Because tungsten has a very high melting temperature $\left(T_{m}=3700 \mathrm{~K}\right)$, deformation experiments performed at room temperature are well into the thermal regime of reduced screw dislocation mobility without the added experimental difficulty of high strain rates $(8)$. In this paper we report the cyclic stress-strain behavior of tungsten monocrystals of a center-triangle orientation and illustrate the behavior in relation to results for other bcc metals.

Additionally, the relationship between the temperature and strain rate dependences of monotonic and cyclic deformation of bcc metals is discussed.

\section{Experimental}

Tungsten single crystals were double- or triple-pass electron-beam zone melted in a vacuum of $<6 \times 10^{-4} \mathrm{~Pa}$ from $5.5 \mathrm{~mm}$ diameter polycrystalline $\mathrm{W}$ bar with a purity of $99.95 \%$. On the last pass the bars were seeded to within $4^{\circ}$ of a [213] rod axis. The bars were subsequently cut to $\sim 70 \mathrm{~mm}$ lengths, centerless ground, electropolished and then annealed in a vacuum of $<1 \times 10^{-6} \mathrm{~Pa}$ at a temperature of $2800 \mathrm{~K}$ for 60 hours. The final cylindrical gage section of the specimens was nominally $10 \mathrm{~mm}$ long and $1.8 \mathrm{~mm}$ in diameter.

Cyclic deformation was carried out using a computer-controlled MTS servohydraulic system described elsewhere (9). Plastic strain control experiments were performed at room temperature at a fixed total strain rate of $8 \times 10^{-5} / \mathrm{s}$ under ambient pressure. Under these conditions $\mathrm{W}$ specimens of a [213] axial orientation exhibit slip behavior in the [1-11] direction and on (011) and (112) planes with slip predominant on different planes in tension and compression (10). Full hysteresis loops were plotted and stored for later analysis.

Some clarification of our testing conditions versus those used by other authors is necessary for later comparisons. Cyclic stress-strain information for $\mathrm{Nb}$ and Mo reported by Guiu and co-workers (2-4) was obtained using a constant total strain amplitude with a manually adjusted total strain rate. Under these conditions the plastic strain amplitude and the peak stresses vary until saturation is reached, complicating any description of cyclic hardening behavior. The plastic strain amplitudes reported in Guiu's work are obtained from the hysteresis loop width at saturation. For Mughrabi's work (5-7), the plastic strain amplitude was controlled, but the strain rate used was a constant 'plastic strain rate', غ́pl, defined as 


\section{$\dot{\varepsilon}$ pl $=2(\Delta \varepsilon p 1) v$}

where $\Delta \varepsilon_{p l}$ is the plastic strain range or twice the plastic strain amplitude,epl, and $v$ is the frequency. An experiment at a true constant plastic strain rate should adjust instantaneously to the changes in slope which occur as the hysteresis loop is traced. The cyclic plastic strain rate defined above requires a set frequency for each plastic strain range, which does not compensate for changes in the elastic strain component of the total strain during cyclic hardening or softening. By using $\dot{\varepsilon}_{p l}$ to control strain rate, much higher values of the conventional total strain rate, $\dot{\varepsilon}$, occur for low plastic strain amplitudes where the elastic/anelastic contribution to hysteresis loop length is greater. Our choice of total strain rate control was dictated by the ease of correlating strain rates between monotonic and cyclic deformation and the knowledge that a total strain rate control parameter adjusts arithmetically to the hysteresis loop length. Thus, as the elastic component of the hysteresis loop increases during cyclic hardening, the time to complete a fatigue cycle correspondingly increases.

\section{Results and Discussion}

The relationship between modulus-compensated saturation stresses and plastic strain amplitude at various homologous temperatures and strain rates for several different bcc metals is shown in Figure 1. The results for tungsten are from our current research and those for other bcc metals are from investigations at similar specimen orientations(2-7). For results from investigations on other bcc metals smooth curves were drawn through data, with the thickness of the lines corresponding approximately to errors in extracting data from published figures. Because no single slip plane is easily defined in cyclic deformation of bcc metals, tensile saturation stresses are shown normalized to Young's modulus. When this method of normalization is used, the cyclic stress-strain data for $W$ and other bcc metals fit a common pattern of temperature and strain rate dependence in spite of detailed differences in experimental procedures. This method of presenting the cyclic stress-strain data provides a global picture of the temperature and strain rate dependences of bcc metals.

Figure 2 shows an idealized, schematic representation of the cyclic deformation data given in Figure 1. Figure 2 suggests that cyclic deformation behavior of bce metals at low homologous temperatures consists of a microstrain-level, low stress plateau and a macrostrain-level, high stress plateau which are connected by a transition region of rapidly increasing saturation stresses as plastic strain amplitude is increased. In general, the extent of the cyclic microstrain region at low plastic strain amplitudes decreases and the plateau of saturation stress which involves macroscopic cyclic deformation at high plastic strain amplitudes increases with decreasing homologous temperature and increasing strain rate. Mughrabi et al. (7) describe deformation at plastic strain levels corresponding to cyclic microstrain as the quasi-reversible motion of an essentially constant density of mobile edge dislocations with very little contribution from screw dislocation motion. The cyclic macrostrain regime is a result of comparable motions of both edge and screw dislocations which evolve into cellular dislocation microstructures. From evaluation of the behavior described in Figure 2, the increased extent of the microstrain regime at higher homologous temperatures is best attributed to an increase in the mobile dislocation density with increasing temperature. Although the relative density of edge dislocations should not change significantly with temperature, the statistical fraction of screw dislocations which have sufficient energy for thermally assisted motion at a given stress level will increase with increasing temperature. At higher temperatures the energy differential between edge and screw dislocation motion in bcc metals becomes small enough to eliminate the upper plateau. The relatively long single plateau in the fatigue of $\mathrm{Cu}$ demonstrates cyclic stress-strain behavior when there is little or no difference in the mobilities of edge and screw dislocations, much like that included for $\mathrm{Mo}$ and $\mathrm{Fe}$ at $\mathrm{T} \pi_{\mathrm{m}} \sim 0.16$ and relatively low strain rates.

If the monotonic microstrain behavior of bcc metals is compared to cyclic microstrain, there are some obvious similarities. For example, the relationship between monotonic microstrain and stress has been shown by Kossowsky in his paper on microyield behavior in Ta (11). When stress versus microstrain relationships are obtained from this data, as in Figure 3 , an increase in the strain hardening rate at strains between the anelastic limit and the eventual macroscopic yield stress is apparent as the temperature is reduced or the strain rate is increased. The anelastic limits are dependent on the prestrain used to separate the true elastic limit and the anelastic limit, which are indistinguishable in fully annealed materials. Additionally, the sensitivity of the measurement technique can influence the results (12). 


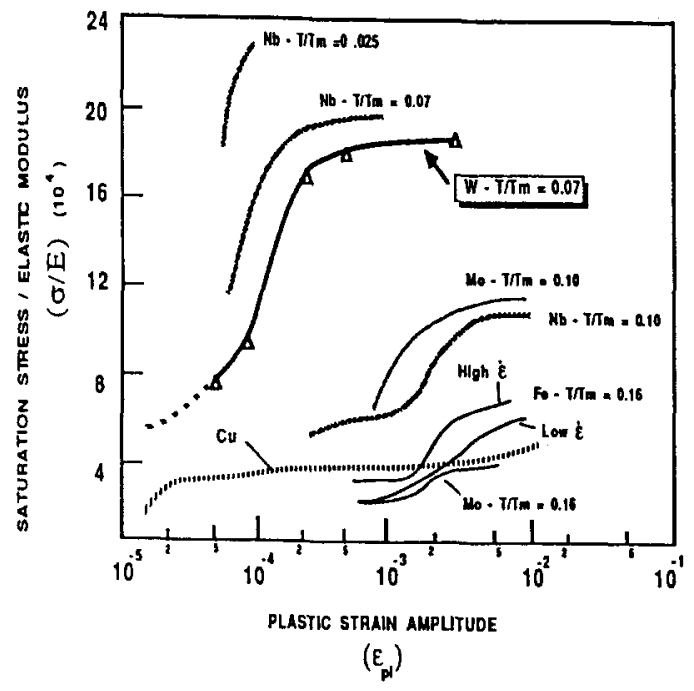

Figure 1. Normalized cyclic stress-strain behavior for bcc metals of center triangle orientations with respect to strain rate and temperature. Data for $\mathrm{Fe}$ is from Mughrabi et al. $(6,7)$. High plastic strain rate $=10^{-2} / \mathrm{s}$; low plastic strain rate $=4.5 \times 10^{-4} / \mathrm{s}$. Data for $\mathrm{Nb}$ and Mo is from Guiu et al. (2). Total strain rate $=6 \times 10^{-4} / \mathrm{s}$.

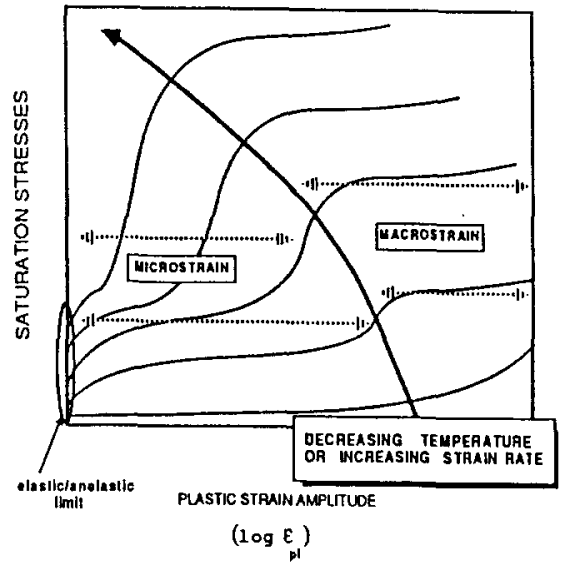

Figure 2. Schematic representation of cyclic stress-strain behavior of bcc metals as a function of temperature or strain rate.

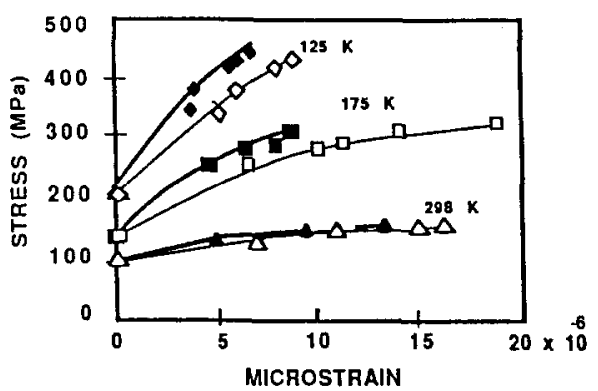

Figure 3. Monotonic microstrain behavior of Ta, adapted from Kossowsky (11). For open symbols total strain rate $=4.3 \times 10^{-4} / \mathrm{s}$; for filled symbols total strain rate $=$ $4.3 \times 10^{-3 / 5}$
Figure 4. Schematic representation of monotonic microflow behavior in a bcc metal as a function of temperature or strain rate. 
However, it is generally accepted that the stress for microstrain is mildly temperature and strain rate dependent, while the stress for macrostrain is strongly temperature and strain rate dependent, as demonstrated by many investigators (1). A schematic representation of the temperature and strain rate dependence of the elastic/anelastic limit, monotonic microflow and macroflow behavior is shown in Figure 4. This behavior can be compared directly with that illustrated in Figure 2 for cyclic microstrain and macrostrain.

Research in our laboratories on surface film softening effects on bcc metals deformed at low homologous temperatures has shown that monotonic microstrain can be extended to larger strain levels through the compatibility-induced generation of mobile edge dislocations at film/substrate interfaces (13). To demonstrate further the relationship between monotonic and cyclic deformation behavior, the temperature dependence of normalized flow stresses for tensile deformation of $W(14)$ and the temperature dependence of normalized saturation stresses for cyclic deformation of bcc metals from the data of Figure 1 are shown respectively in parts (a) and (b) of Figure 5. Also shown in Figure 5(a) is the relative insensitivity of flow stresses to temperature when edge dislocation motion dictates monotonic plastic flow. Comparison of this behavior to the relatively limited temperature dependence of saturation stresses during cyclic microstrain shown in Figure 5(b) illustrates the similar temperature dependent response of bcc metals to monotonic and cyclic deformation at both microstrain and macrostrain levels of plastic flow.

\section{Acknowledgements}

This research is supported by the National Science Foundation, DMR-8506705.

\section{References}

1 J. W. Christian, Met. Trans. 14A, 1237 (1983).

2. F. Guiu, J. A. Planell and M. Anglada, in Dislocations in Real Materials, ed. M. H. Loretto, p. 263, Institute of Metals, (1985).

3. M. Anglada B. Etemad, J. A. Planell and F. Guiu, Scripta Met. 14, 1319 (1980).

4. M. Anglada and F. Guiu, Phil. Mag. 44, 499 (1981).

5. H. Mughrabi, in Dislocations in Real Materials, ed. M. H. Loretto, p.244, Institute of Metals, (1985).

6. H. Mughrabi, K. Herz and X. Stark, Int. J. of Fracture 17, 193 (1981).

7. H. Mughrabi, K. Herz and X. Stark, Acta Met., 24, 659 (1976).

8. J. R. Stephens, Reviews on The Deformation Behavior of Materials 1, 32 (1974).

9. D. S. Grummon, Ph.D. Thesis, The University of Michigan, Ann Arbor, Michigan, (1986)

10. K. J. Bowman, Ph.D. Thesis, The University of Michigan, Ann Arbor, Michigan, in progress.

11. R. Kossowsky, in Refractory Metals and Alloys IV, Met. Society Conf. Series, 41, p. 47 (1965).

12. J. D. Meakin, Canadian J. Physics 45, 1121 (1967).

13. R. D. Noebe and R. Gibala, in Phase Boundary Effects in Mechanical Behavior, ed.

K. Subramanian and I. A. Iman, TMS, Warrendale, Pennsylvania, in press.

14. J. E. Talia, Ph.D. Thesis, Case Western Reserve University, Cleveland, Ohio, (1981).
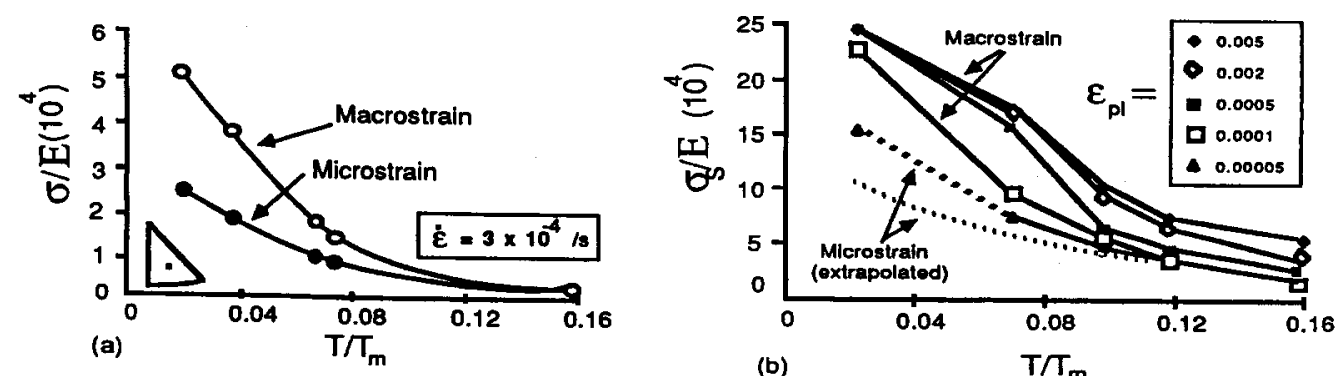

Figure 5. (a) The temperature dependence of microstrain-level and macrostrain-level flow stresses for $W$ single crystals of a [213] axial orientation. Data obtained from surface film softening experiments (14). (b) The temperature dependence of cyclic microstrain and cyclic macrostrain saturation stresses in the cyclic deformation of bcc metals adapted from the data of Figure 1. 